\title{
Cortical potentials evoked by a TV pattern reversal stimulus with varying check sizes and stimulus field
}

\author{
G. BARTL, * G. H. M. VAN LITH, AND G. W. VAN MARLE \\ From the Eye Hospital, Erasmus University, Rotterdam
}

SUMMARY With the use of television equipment the influence of check size and stimulus field on the pattern evoked cortical potentials was investigated. Maximum responses were found with $20^{\prime}$ and $40^{\prime}$ checks. The major part of the responses was initiated from a ring between $1 \cdot 25^{\circ}$ and $2 \cdot 5^{\circ}$ eccentricity, though relatively per stimulus area the response became progressively larger the smaller the stimulus field.

Visually evoked cortical potentials (VECPs) can be elicited either by spatial unstructured stimuli or by patterned stimuli. The latter can be divided into flashed pattern, pattern appearance, pattern disappearance, and pattern reversal stimuli. Pattern responses are generally larger than those evoked by unstructured stimuli. The reason for this is that they are not only a luminance response but also a contrast response. Since in pattern reversal stimuli the luminance of the entire stimulus field is constant, the response will mainly be specific for contrast.

Stimulators for pattern reversal, like the ones used by Riggs et al. (1966) or Spekreijse (1966), are rather complicated for routine examinations. Pattern stimulation according to the method of Cobb et al. (1967) or by means of Polaroid plates (Behrman et al., 1972) are simpler but are not versatile. More promising seems to be pattern stimulation by a television set, recently introduced by Arden and Faulkner (1977). It combines simplicity with variability. From the experience obtained with a pattern stimulus like the one used by Cobb, a TV set for pattern stimulation was adjusted by one of us (van Marle and van Lith, 1976).

With pattern stimulation 2 main questions are important, namely, the influence of the check size on the VECPs and the effect of the size of the stimulus field. These questions have already been investigated by Spekreijse (1966), Regan and Richards (1971), and Harter (1971). However, a pattern on a TV screen does not have the same contour sharpness as

* Visiting scientist from the University Eye Hospital, Graz, Austria

Address for reprints: Mr G. H. M. van Lith, Electroophthalmological Department, Eye Hospital, Schiedamse Vest 180, 3000 LM Rotterdam, The Netherlands that obtained by other methods, so that these experiments had to be repeated with the TV pattern.

\section{Method}

Four healthy subjects with normal visual acuity and normal visual fields, the latter determined by static perimetry, were examined. The black-and-white monitor has a screen of $48 \times 38 \mathrm{~cm}$. The stimulator produces a chessboard pattern on the screen. Check sizes are adjustable in 4 steps, while the modulation depth is variable from 0 to $98 \%$. The luminance of the monitor's screen can be varied between 10 and 500 asb.

The subjects sat $2 \mathrm{~m}$ in front of the monitor. Only 1 eye was examined, the other being closed. A point had to be fixated in the centre of the TV screen. For the experiments described here a pattern reversal stimulus was applied. The frequency was 4.9 cycles $/ \mathrm{s}$ and the modulation depth $20 \%$. Check sizes of $10^{\prime}, 20^{\prime}, 40^{\prime}$, and $80^{\prime}$ visual angle were used. The stimulated area was varied by covering parts of the screen with black paper. In this way stimulus areas of $1 \cdot 25^{\circ}, 2 \cdot 5^{\circ}, 5^{\circ}, 7 \cdot 5^{\circ}$, and $10^{\circ}$ visual angle could be obtained. In addition, full field stimulation (FF)-that is, the entire screen of the TV set-was applied. The latter represented a visual angle of $11^{\circ}$ in the vertical direction and of $14^{\circ}$ in the horizontal direction. In another series the reverse was done by covering the centre of the screen according to the above-mentioned visual angles. Each subject was examined at 3 sessions on different days and twice in each session.

Four scalp electrodes in the midline at 5, 15, 25, and $35 \%$ above the inion were used with reference to the ear lobe. The electrode position which gave the highest response was used for the experiments. 


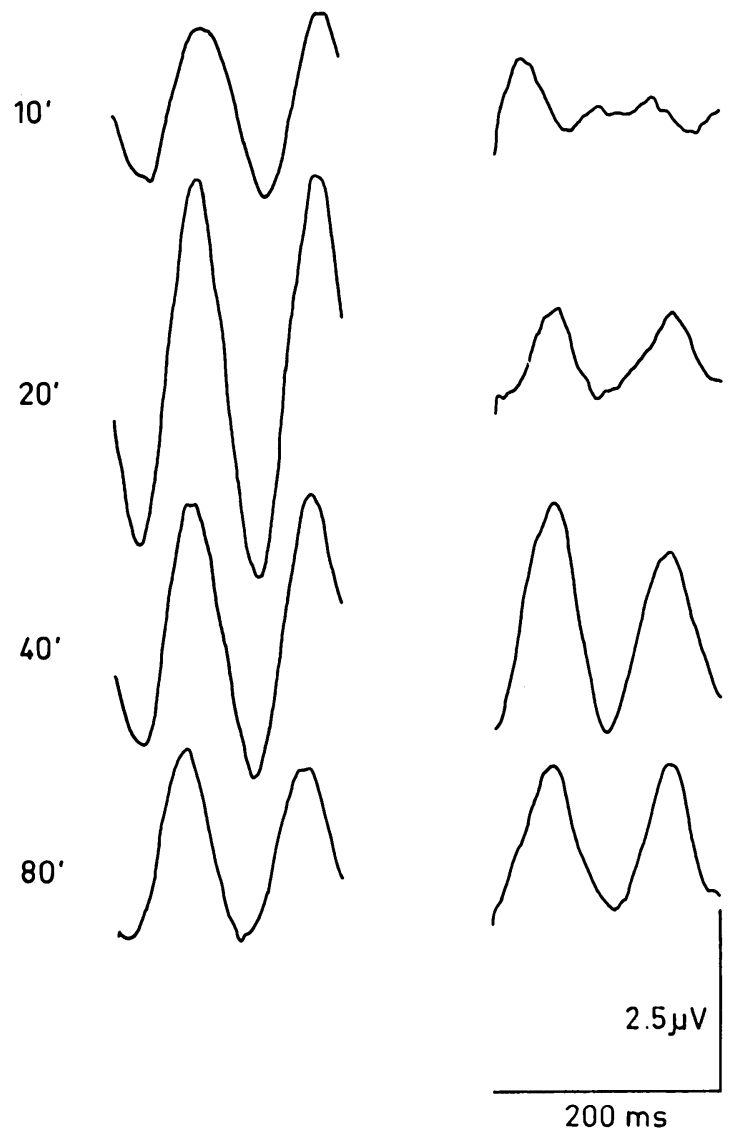

Fig. 1 Recordings of pattern reversal evoked potentials of Subject 3 with checks of 10',20',40', and 80'. The evoked potentials in the left column were obtained with a centrally-viewed stimulus field of $7 \cdot 5^{\circ}$ visual angle, and the right column shows evoked potentials in the reverse situation, namely $7 \cdot 5^{\circ}$ visual angle of the TV screen covered

In Subjects 1 and 2 this was at the $15 \%$ position above the inion and in Subjects 3 and 4 at the $5 \%$ position. After amplification 256 signals were averaged by a Datalab $102 \mathrm{~S}$, of which the analysis time was set at $200 \mathrm{~ms}$. An active filter was applied, tuned to $9 \cdot 8 \mathrm{~Hz}$ (Q-factor $3 \cdot 5$ ). The registration was done with an X-Y plotter.

\section{Results}

In preliminary experiments no change was observed if the distance to the monitor was increased from 1 to $5 \mathrm{~m}$, the visual angle of the check size being kept constant. Fig. 1 represents an example of the actual recordings, while in Figs. 2 and 3 the amplitude of the responses is plotted against the check size.
The parameter is the stimulus field. Each point represents the mean value of 6 examinations. In order to make our diagrams less complicated the curves have been drawn without the standard errors (SE). Those of the maxima of the centrally-viewed stimuli (Fig. 2) are given in the Table below. These maxima were obtained in Subjects 1 and 2 with the $40^{\prime}$ check size and in Subjects 3 and 4 with the $20^{\prime}$ check size.

\begin{tabular}{lllll}
\hline \multirow{2}{*}{ Subject } & \multicolumn{4}{l}{ Stimulus field } \\
\cline { 2 - 5 } & $5^{\circ}$ & $7.5^{\circ}$ & $10^{\circ}$ & $f f$ \\
\hline 1 & 1.44 & 1.18 & 2.02 & 1.57 \\
2 & 0.95 & 0.71 & 1.53 & 0.94 \\
3 & 0.31 & 0.40 & 0.28 & 0.58 \\
4 & 1.11 & 1.68 & 1.14 & 0.98 \\
\hline
\end{tabular}

In the graphs of Fig. 2 an increase in response can be seen when the size of the stimulus field is increased. With the stimulus field of $1 \cdot 25^{\circ}$ all subjects have responses smaller than $2 \mu \mathrm{V}$, while the check size has no influence. With a stimulus field of $2 \cdot 5^{\circ}$ the results are about the same for Subjects 1 and 2 but not for Subjects 3 and 4 ; and of the latter two the responses are somewhat higher with a check size of $20^{\prime}$ (Subject 3: $2 \cdot 3 \mu \mathrm{V}$, and Subject 4: $4.8 \mu \mathrm{V})$. A marked increase in response in all subjects is observed with a stimulus field of $5^{\circ}$. The responses are now also clearly dependent on the check size. While Subjects 1 and 2 have a maximal response with a check size of $40^{\prime}(5.6 \mu \mathrm{V}$ and $5.7 \mu \mathrm{V})$, Subjects 3 and 4 have their maximal response with a check size of $20^{\prime}(3.7 \mu \mathrm{V}$ and $7.4 \mu \mathrm{V})$. Furthermore, it may be observed that the maximum response of Subjects 1 and 3 increases regularly when the stimulus field is enlarged, whereas in Subjects 2 and 4 the highest amplitude is noticed with the stimulus field of $7 \cdot 5^{\circ}$, smaller amplitudes are obtained with a stimulus field of $10^{\circ}$.

Fig. 3 shows that, if instead of the periphery the centre of the TV screen was covered by targets of various diameters, the results were in all subjects the reverse of those shown in Fig. 2. The responses remained good when a central area of $1.25^{\circ}$ or $2.5^{\circ}$ was covered, while they became much smaller with a black central target of $5^{\circ}, 7^{\circ}$, and $10^{\circ}$.

Comparing Figs. 2 and 3 shows that the maxima of the peripheral responses tend to occur at larger check sizes than those of the central responses.

To find out the response per retinal location, the ratio between the height of the response and the surface area has been calculated. This has been done only for those check sizes which produced the highest response-hence in Subjects 1 and 2 for check size $40^{\prime}$ and in Subjects 3 and 4 for check 

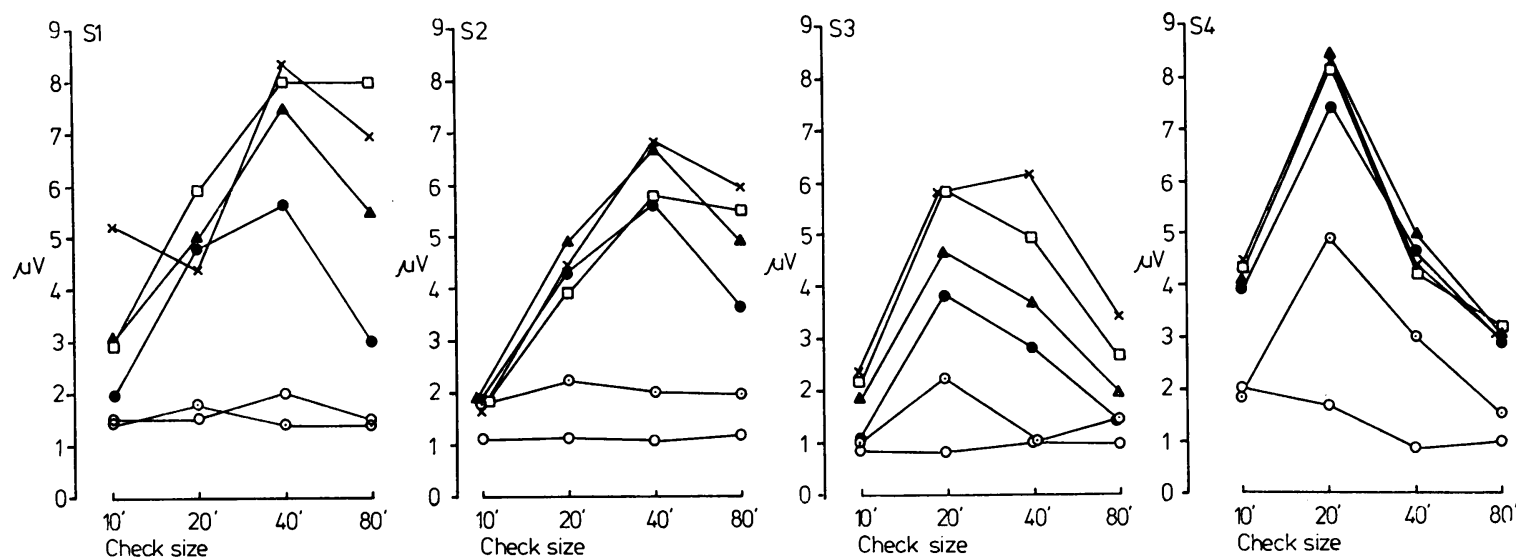

Fig. 2 Pattern reversal evoked potentials of Subjects 1-4 obtained with centrally-viewed stimulus fields of $1 \cdot 25^{\circ}$ $(\bigcirc-O) ; 2.5^{\circ}(\odot-\bigcirc) ; 5^{\circ}\left(\bigcirc_{-}\right) ; 7.5^{\circ}(\triangle-\triangle)$; and $10^{\circ}(\square-\square)$ visual angle and full field $(\times-\times)$ plotted against check size. Electrodes were placed in the midline at $15 \%$ in Subjects 1 and 2 and at $5 \%$ in Subjects 3 and 4 , the reference electrode being on the ear lobe. Each point on the graphs is the mean value of 6 examinations
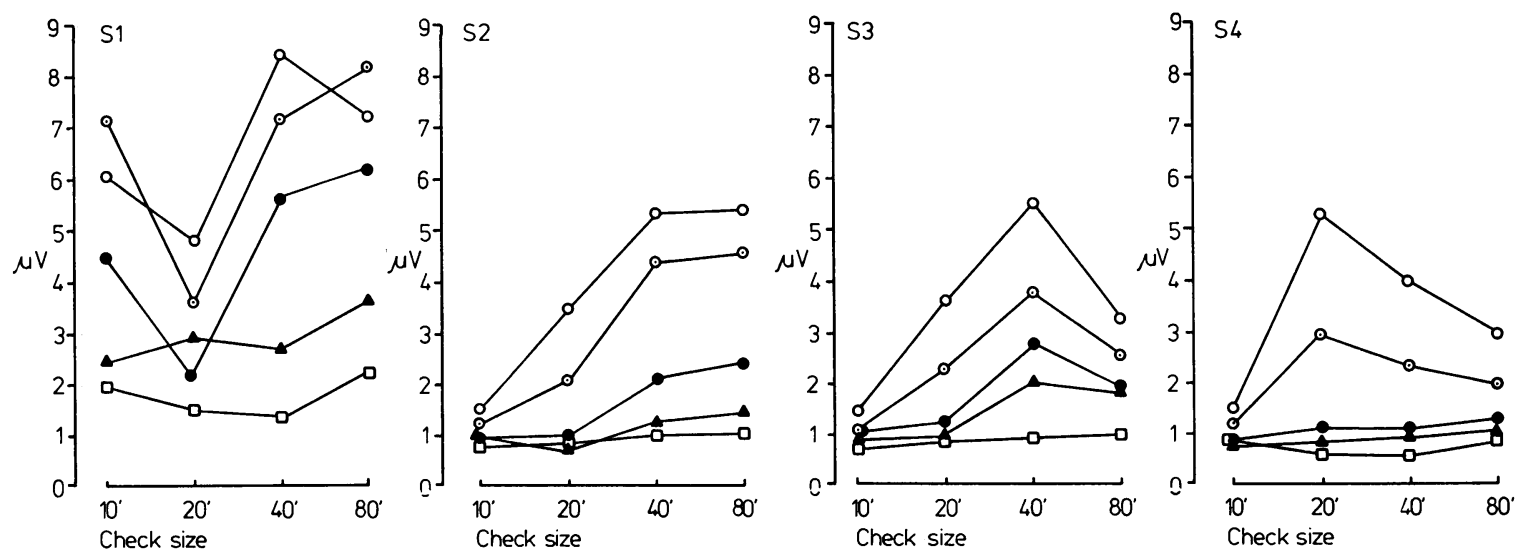

Fig. 3 Pattern reversal evoked potentials of Subjects 1-4 obtained with peripheral stimulus fields. The centre was covered by black targets of $1 \cdot 25^{\circ}, 2 \cdot 5^{\circ}, 5^{\circ}, 7^{\circ}$, and $10^{\circ}$ visual angle. The other parameters are the same as described in Fig. 2

size $20^{\prime}$. In Fig. 4 these ratios have been plotted as a function of the stimulus field. The responses obtained with a stimulus field of $1.25^{\circ}$ and $2.5^{\circ}$ were too small for evaluation and therefore were excluded from the diagram. For all subjects the response per surface area decreased with increasing stimulus field, hence also with increasing mean eccentricity of the stimulus field.

\section{Discussion}

In general it may be stated that with our TV set centrally-viewed stimulus fields smaller than $2.5^{\circ}$ and check sizes smaller than $20^{\prime}$ produce VECPs below the $2 \mu \mathrm{V}$ level. Maximum responses were found with $20^{\prime}$ and $40^{\prime}$ check sizes. These results are not in accordance with those of Spekreijse (1966), who described well-defined VECPs with stimulus fields smaller than $1.9^{\circ}$ and with a check size of $11^{\prime}$. Nor are they in accordance with those of Regan and Richards (1971), who found maximum responses between $11^{\prime}$ and $18^{\prime}$ check size. This discrepancy can probably be attributed either to intersubject variability or to differences in the stimulus technique. A TV pattern has less contour sharpness in comparison with Cobb and Morton's projection method and Spekreijse's mirror apparatus. Another distinction between these apparatuses and the TV set is that in the latter the reversal movement of the pattern is sequential in time. This 
Fig. 4 Amplitudes per square centimetre of stimulus area plotted against stimulus fields of $5^{\circ}, 7 \cdot 5^{\circ}, 10^{\circ}$, and full field from Subject 2 - - 0 ; Subject 3 $\Delta-\mathbf{\Delta}$; Subject $4 \mathrm{O}-\mathrm{O}$ ) in relation to the check size giving the highest evoked potentials (Subjects 1 and 2 at 40' and Subjects 3 and 4 at 20') Subjects 1-4 (Subject $10-\bigcirc$;

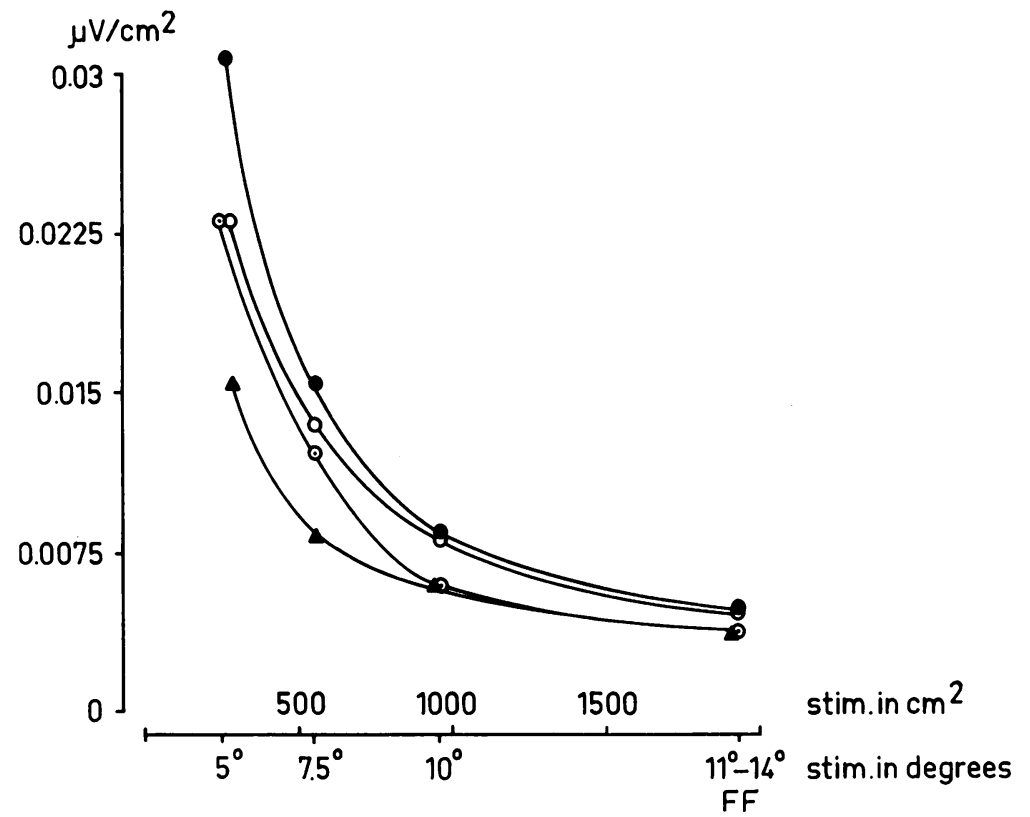

is caused by the line speed of the frame and amounts to $20 \mathrm{~ms}$. We tried to increase the contour sharpness by increasing the distance between the TV set and the subject from 1 to $5 \mathrm{~m}$, keeping the check size and field size constant. This causes the lines of the TV screen to fall below the minimum separable. There was no clear effect.

Measured absolutely, the major part of the response in our set-up is initiated from a ring between $1.25^{\circ}$ and $2.5^{\circ}$ eccentricity, since the response increased most when going from a $2.5^{\circ}$ stimulus field to a $5^{\circ}$ stimulus field, changing less below $2 \cdot 5^{\circ}$ and above $5^{\circ}$ (Fig. 2). The response per stimulus area, however, becomes progressively larger the smaller the stimulus field (Fig. 3). The latter is consistent with the correlation between the amplitude of luminance VECPs for small stimulus fields and the number of cones stimulated, as found by van Lith and Henkes (1970).

With respect to the VECPs produced by peripheral stimulus fields, in 3 subjects a maximum was obtained with larger checks than by central stimulation. This was also found by Harter (1971) using flashed pattern stimulation. The insensitivity of the extrafoveal region to small checks is probably related to the decrease in cone density and the convergence of the extrafoveal cones into the ganglion cells. A relative insensitivity of the central fovea to larger checks is explained if the checks cover the entire central fovea. A luminance response instead of a contrast response will then be obtained.

\section{References}

Arden, G. B., and Faulkner, D. J. (1977). A versatile pattern generator for neuro-ophthalmological and paediatric EP and psychophysical tests, using standard television techniques compatible with broadcast colour programmes. In Experimental and Clinical Amblyopia. Proceedings XIIIth Iscerg Symposium, Israel 1975. Pp. 165-166. Documenta Ophthalmologica Proceedings Series 11. Edited by E. Auerbach.

Behrman, J., Nissim, S., and Arden, G. B. (1972). A clinical method for obtaining pattern visual evoked responses. In The Visual System: Neurophysiology, Biophysics, and their Clinical Applications; Proceedings IX Iscerg Symposium, Brighton 1971. Pp. 199-206. Edited by G. B. Arden. Plenum Press: New York.

Cobb, W. A., Morton, H. B., and Ettlinger, G. (1967). Cerebral potentials evoked by pattern reversal and their suppression in visual rivalry. Nature, 216, 1123-1125.

Harter, M. R. (1971). Evoked cortical responses to checkerboard patterns: effect of check size as a function of retinal eccentricity. Vision Research, 10, 1365-1376.

Lith, G. H. M. van, and Henkes, H. E. (1970). The relationship between ERG and VER. Ophthalmic Research, 1, 40-47.

Marle, G. W. van, and Lith, G. H. M. van (1976). Monitor als Musterstimulator für visuell evozierte Potentiale (VEP). In Wissenschaftliche Vorträge, 1. Pp. 6-10. Jahrestagung Österreichische Gesellschaft für biomedizinische Technik, Graz.

Regan, D., and Richards, W. A. (1971). Independence of evoked potentials and apparent size. Vision Research, 11, 679-684.

Riggs, L. A., Johnson, E. P., and Schick, A. M. L. (1966). Electrical responses of the human eye to changes in wavelength of the stimulating light. Journal of the Optical Society of America, 56, 1621-1627.

Spekreijse, H. (1966). Analysis of EEG responses in man. Thesis. Junk: The Hague. 